\title{
Mechanical Reinforcement of Polymeric Fibers through Peptide Nanotube Incorporation
}

\author{
Daniel J. Rubin, ${ }^{\dagger, \dagger}$ Hadi T. Nia, ${ }^{\S}$ Thierry Desire, $"$ Peter Q. Nguyen, ${ }^{\dagger, \ddagger}$ Michael Gevelber, \\ Christine Ortiz, ${ }^{\S}$ and Neel S. Joshi ${ }^{*, t, \$}$ \\ ${ }^{\dagger}$ School of Engineering and Applied Sciences, Harvard University, Cambridge, Massachusetts 02138, United States \\ ${ }^{\ddagger}$ Wyss Institute for Biologically Inspired Engineering, Boston, Massachusetts 02115, United States \\ ${ }^{\S}$ Department of Materials Science and Engineering, Massachusetts Institute of Technology, Cambridge, Massachusetts 02139, United \\ States \\ "Department of Mechanical Engineering, Boston University, Boston, Massachusetts 02215, United States
}

\section{Supporting Information}

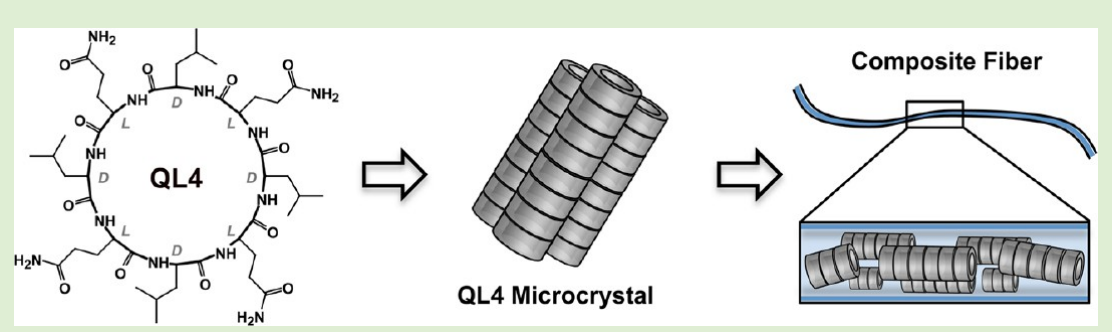

ABSTRACT: High aspect ratio nanotubular assemblies can be effective fillers in mechanically reinforced composite materials. However, most existing nanotubes used for structural purposes are limited in their range of mechanical, chemical, and biological properties. We demonstrate an alternative approach to mechanical reinforcement of polymeric systems by incorporating synthetic D,L-cyclic peptide nanotube bundles as a structural filler in electrospun poly D-, L-lactic acid fibers. The nanotube bundles self-assemble through dynamic hydrogen bonding from synthetic cyclic peptides to yield structures whose dimensions can be altered based on processing conditions, and can be up to hundreds of micrometers long and several hundred nanometers wide. With 8 wt \% peptide loading, the composite fibers are $>5$-fold stiffer than fibers composed of the polymer alone, according to atomic force microscopy-based indentation experiments. This represents a new use for self-assembling cyclic peptides as a load-bearing component in biodegradable composite materials.

\section{INTRODUCTION}

Materials whose mechanical and physical properties can be precisely tuned are critical for the fabrication of effective tissue engineering scaffolds, ${ }^{1}$ wound dressings, ${ }^{2,3}$ medical sutures, filtration devices, and textiles. ${ }^{4}$ Increasingly, a composite design in which a high aspect ratio filler material is incorporated within a less-structured matrix is employed in order to match the specific combination of high-performance properties required by the structure. For example, carbon nanotubes $(\mathrm{CNTs})^{5}$ and cellulose nanowhiskers ${ }^{6}$ have been investigated extensively to increase the stiffness and strength of synthetic polymer fibers. Mathematical models and empirical studies of composite materials have suggested that there are four key factors that affect their mechanical properties: (1) the Young's modulus of the filler material, (2) the strength of the molecular interactions between the filler material and the surrounding polymer matrix, (3) the filler material aspect ratio, and (4) the orientation of the supporting material with respect to the fiber long axis. ${ }^{7,8}$ Both CNTs and cellulose nanowhiskers make for effective filler materials in some composites because they exhibit high Young's moduli and high aspect ratios. However, due to their methods of synthesis, they both exhibit limited capabilities in terms of controlling the spatial complexity of possible surface modifications, accessing a wide range of dimensions, and inducing assembly and disassembly. Both materials are generally regarded as nonbiodegradable, raising questions about their ultimate fate after incorporation into biological systems. ${ }^{9}$ Although cellulose nanowhiskers are thought to be biocompatible, toxicity remains an ongoing hurdle for CNTs. ${ }^{10}$

Materials created through self-assembly may provide alternative reinforcement systems that exhibit a combination of biocompatibility and biodegradability, enable the formation of nanostructures with controllable dimensions, allow for rapid exploration of complex surface chemistries, and provide refined methods for stimulus responsive and self-healing materials. Specifically, due to their straightforward synthesis and customizability, ${ }^{11}$ engineered peptides have emerged as a powerful way to create nanostructures and larger scale materials with highly tunable properties. ${ }^{12}$ However, the vast majority of these systems are designed such that short linear amino acid

Received: June 7, 2013

Revised: September 18, 2013

Published: September 26, 2013 


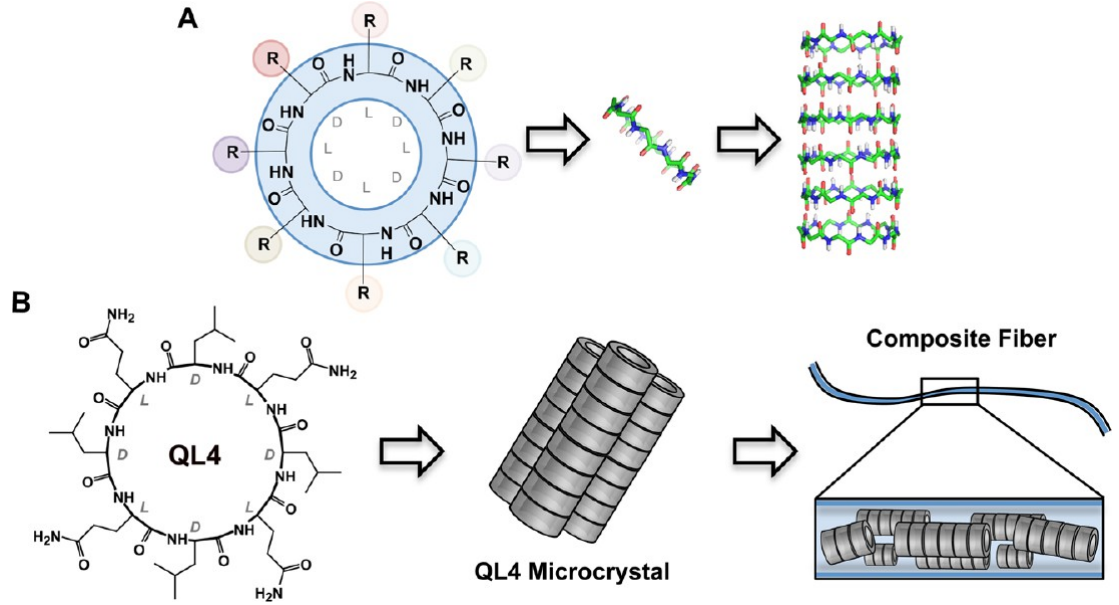

Figure 1. (A) Schematic of an 8-amino acid D,L-cyclic peptide. $R=$ side chain. Individual monomers stack atop one another in antiparallel $\beta$-sheets, resulting in peptide nanotubes and larger crystals. (B) Chemical structure of QL4, schematic of QL4 microcrystal, and QL4-Polymer composite structure.

sequences assemble to form porous networks composed of entangled supramolecular fibers. ${ }^{13,14}$ In order to create peptidebased filler materials that can mimic the mechanical stability of conventional fillers, a supramolecular system capable of forming rigid assemblies is required.

We have developed a bottom-up approach to synthesizing filler materials for composite reinforcement based on the selfassembly of D-, L-cyclic peptides (DLCPs). Originally selected for use as membrane-disrupting antibiotics due to their unique structure, customizable surface chemistry, and potential for biocompatibility, ${ }^{15}$ DLCPs have since been underutilized in biomaterials applications. The peptide cycles are composed of eight amino acids with alternating D- and L-stereochemistry, causing a planar geometry in which the amino acid side chains radiate from the center of the ring and the amide backbone is perpendicular to the plane of the ring, promoting their assembly into high aspect ratio nano- and microstructures through $\beta$-sheet-like hydrogen bonding (Figure 1A). ${ }^{16}$ Depending on the sequence of the peptides, the nanotubes may also associate laterally into bundles to create structures that can be hundreds of micrometers in length. Although the Young's modulus of DLCP assemblies has yet to be determined directly using nanomechanical characterization techniques, they are estimated to be comparable to the stiffest known self-assembled organic systems. ${ }^{17}$ Thus, the self-assembled structures exhibit many of the desirable structural features for filler materials in composites: they are mechanically rigid, they have high aspect ratios, and their surface chemistry can be customized to maximize filler-matrix interactions. Most importantly, since DLCP nanotubes are created through bottom-up self-assembly, they represent an intriguing dynamic scaffold whose physical properties can be changed based on assembly state, allowing for future work in stimulus responsive and self-healing materials.

We demonstrate the mechanical reinforcement of synthetic polymer fibers using DLCP-NTs by atomic force microscopy (AFM)-based indentation experiments. This was accomplished through the fabrication of composite fibers where the major component was poly(D-, L-lactic acid) (PDLLA) and the minor component was DLCP-NTs (Figure 1B). While aliphatic polyesters such as PDLLA are convenient to use because they are commercially available and have been deemed safe for human implantation by the FDA, their mechanical and physical properties are suboptimal for applications in which they must replace or augment load-bearing tissues. ${ }^{18}$ PDLLA was selected for this study because (1) it is commonly used in the fabrication of implantable biomedical materials, ${ }^{4}$ (2) its amorphous structure prevents the creation of nanocrystalline irritants upon biodegradation, ${ }^{19}$ yet (3) it is less stiff than its crystalline analogues, and (4) it is unstable when subjected to a static load. ${ }^{20}$ Despite the extensive study of self-assembled peptide systems for various applications, there have been few examples $^{21}$ of their use as mechanical reinforcement agents. We have accomplished the controlled synthesis of DLCP-NT bundles, their incorporation into electrospun PDLLA fibers, and the mechanical characterization of this system. This represents the first demonstration of DLCPs as mechanically stabilizing components of a composite material.

\section{EXPERIMENTAL SECTION}

Chemicals and Reagents. Acetone, dichloromethane, dimethylformamide, $N, N$-diisopropylethylamine (DIPEA), and piperidine were purchased from Sigma Aldrich. Dichloromethane and dimethylformamide were dried over molecular sieves. The following chemicals were used as provided: Acetone, trifluoroacetic acid, O- $(1 \mathrm{H}-6$-chlorobenzotriazole-1-yl)-1,1,3,3-tetramethyluronium hexafluorophosphate (HCTU), tetrakis(triphenylphosphine)palladium (TPP-Pd), phenylsilane, and (benzotriazol-1-yl-oxytripyrrolidinophosphonium hexafluorophosphate) (РyBOP) (Sigma Aldrich). All amino acids and Rink Amide-MBHA resin were purchased from AAPPTEC, Louisville Kentucky.

Cyclic Peptide Synthesis. D,L-cyclic peptides were synthesized in accordance with the procedure of McMurray. ${ }^{22}$ Fmoc-Glu-OAll was coupled to a Rink Amide-MBHA resin through the side-chain carboxylate. When cleaved, this residue is converted to a Gln. Standard Fmoc synthesis produced an uncyclized 8-mer. The protecting allyl group was removed through two successive 1-hour incubations with TPP-Pd and phenylsilane under argon. The product was then cyclized by two 12-hour rounds of a PyBOP-assisted coupling reaction. Peptides were cleaved from the resin with $95 \%$ trifluoroacetic acid (TFA), $2.5 \%$ water, and $2.5 \%$ triisopropylsilane. To isolate the peptide, the TFA solution was concentrated by evaporation and dropped into cold diethyl ether causing precipitation. The mixture was centrifuged, resuspended in TFA, and precipitated again to increase purity. Cyclic peptide identity was verified by liquid chromatography electrospray ionization mass spectroscopy.

Self-Assembly. Self-assembly was achieved by dissolving $2.5 \mathrm{mgs} /$ $\mathrm{ml}$ of QL4 in a mixture of $60 \%$ TFA and $40 \%$ water. The assembly 
occurred in a glass vial over $48-72 \mathrm{~h}$ at which point microcrystals could be seen by eye. Crystals were harvested by diluting the assembly with a mixture of acetone and dichloromethane and pelleting the crystals by centrifugation. Crystals were rinsed 3 times with acetone/ dichloromethane before characterization and incorporation in PDLLA.

Electrospinning. Nanofibers were produced by traditional electrospinning methods. Solutions containing $8 \%$ poly-D,L-lactide and varying amounts of QL4 microcrystals ranging from $0 \mathrm{wt} \%$ to $8 \mathrm{wt}$ $\%$ in 3:1 acetone to dichloromethane were vortexed to promote mixing. The solutions were then electrospun at a flow rate of $0.07 \mathrm{~mL} /$ min, $25 \mathrm{kV}$ and at $30 \mathrm{~cm}$ from the collecting plate. Samples were collected on aluminum foil and silicon wafers and stored for later use.

Field Emission Scanning Electron Microscope (FE-SEM) Imaging of Fibers and DLCP Nanotubes. Electrospun fiber samples deposited on aluminum foil were cut and mounted onto SEM stubs using carbon adhesive. For the recovery of DLCPs from nonwoven mats, nanofibers were incubated in acetone, selectively dissolving the polymer while leaving QL4 microcrystals intact. Once the PDLLA had dissolved, QL4 was pelleted by centrifugation and washed three times with acetone, before resuspension in water. This suspension was applied to a Nuclepore filter under low vacuum, the filters were allowed to dry under vacuum, and then mounted onto SEM stubs as above. All samples were sputter-coated with $\mathrm{Au} / \mathrm{Pd}$ and then imaged on a Zeiss FE-SEMSupra55VP (Carl Zeiss, Oberkochen, DE) in SE2 mode.

Fourier-Transform Infrared Spectroscopy. A Bruker Hyperion 3000 FTIR microscope (Bruker Optics Inc., Billerica, MA) was used to confirm the presence of DLCPs in the composite fibers. Electrospun fibers, with aluminum foil backing, were placed under the objective and measurements were taken in reflectance mode.

Mechanical Characterization. An Asylum MFP3D AFM (Asylum Research, Santa Barbara, CA) was used to perform the nanoindentation on single fibers atop a silicon wafer in the dry state. A glass spherical probe tip (Polysciences, Warrington, PA), diameter $\sim 40 \mathrm{um}$, was attached to the tipless cantilever with nominal spring constant $k \sim 30 \mathrm{~N} / \mathrm{m}$ (Budget Sensors, Sofia, Bulgaria). The thermal oscillation method was applied to determine the cantilever spring constant for each probe tip. ${ }^{23}$ The indentation was performed under force control scheme, with maximum force $\sim 900 \mathrm{nN}$. The axial $(z$ direction) displacement of tip is calculated as the $z$-piezo subtracted by the vertical deflection of the cantilever. All data reported in this manuscript is based on axial loading of fibers.

\section{RESULTS AND DISCUSSION}

DLCPs were synthesized on the solid phase using previously published protocols. ${ }^{16}$ For this study, we used cyclo-[(QL $\left.)_{4}\right]$, which we abbreviate as QL4 (Figure S1, Supporting Information). This sequence was chosen because of its known tendency to assemble into large, stable bundles of tubes, which here we call microcrystals. ${ }^{16}$ In this case, assembly was accomplished by dissolving the DLCPs in TFA and adding pure water until a concentration of 40:60 water was reached. Formation of DLCP-NT bundles (microcrystals) proceeded over the course of $48 \mathrm{~h}$. SEM analysis of the microcrystals revealed that they exhibited a range of dimensions. The largest observed structures had diameters of several hundred nanometers and reached $10-50 \mu \mathrm{m}$ in length (Figure 2A). Given that a single nanotube of stacked DLCPs is only a nanometer in diameter, this indicates that the microcrystals are composed of hundreds of laterally associated DLCP-NTs. Previous reports of QL4 assemblies have suggested that this bundling is mediated by hydrogen bonding between glutamine residues. ${ }^{16}$ Importantly, the microcrystals were quite robust once assembled, being able to withstand centrifugation and vortexing. However, upon ultrasonication the microcrystals were shortened, presumably by transverse fracture, since the average lengths decreased from $14 \pm 12 \mu \mathrm{m}$ to $3 \pm 2 \mu \mathrm{m}$ while the average
A

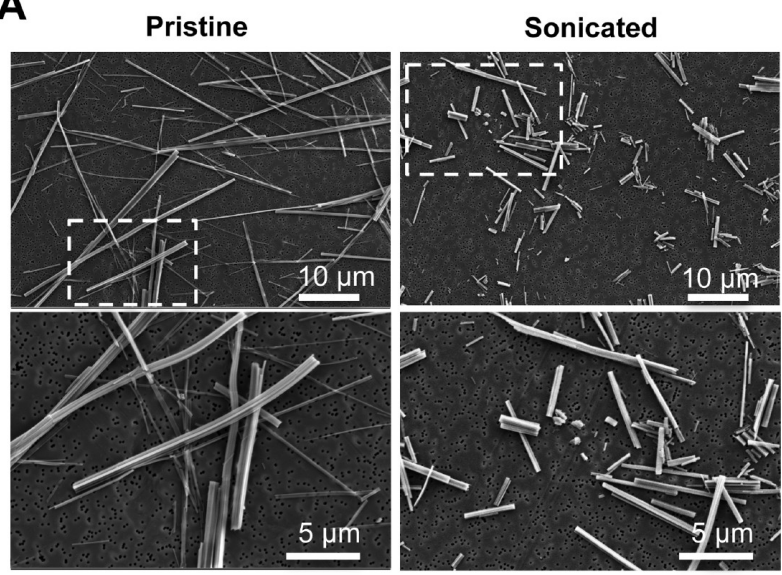

B

Length of QL4 Microcrystals

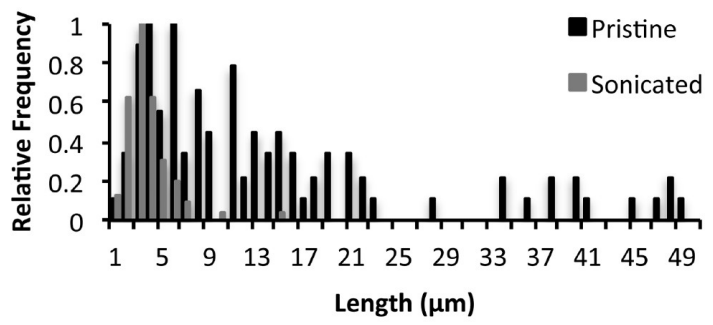

Diameter of QL4 Microcrystals

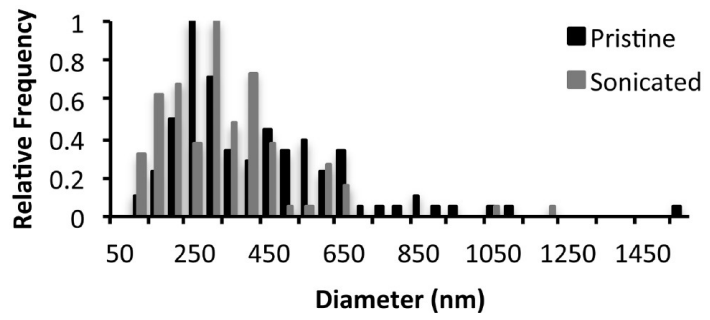

Figure 2. Size characterization of QL4 microcrystals (bundles of DLCP-NTs). (A) SEM images of microcrystals before and after sonication. White dashed boxes indicate region that is magnified in the lower images. (B) Length and width distributions of microcrystals.

widths remained largely unchanged (Figure 2B). Lyophilization also led to DLCP-NT degradation to varying degrees (Figure S2).

To prepare the spinning solution, the DLCP-NTs were mixed with PDLLA in 3:1 acetone/dichloromethane. As QL4 is generally hydrophobic, yet has a high density of hydrogen bonding moieties, it was expected to interact favorably with PDLLA. In order to probe the dispersion of DLCPs into the polymer matrix, droplets of the spinning solution were placed on glass slides and allowed to dry. Optical micrographs of the dried droplets revealed that the intact DLCP-NTs at $1 \%, 4 \%$, and $8 \%$ loading were evenly dispersed inside the polymer matrix (Figure S3). In contrast, DLCP-NTs that were disassembled and aggregated did not disperse evenly and formed clumps within the dried spinning solution (Figure S3).

Electrospinning experiments confirmed that composite nanofibers could be obtained with varying concentrations of PDLLA and microcrystals. A noteworthy observation is that solutions containing pure PDLLA formed lightly beaded fibers at and below a polymer concentration of $8 \%$ by weight (Figure S4). However, when QL4 microcrystals were added to the 


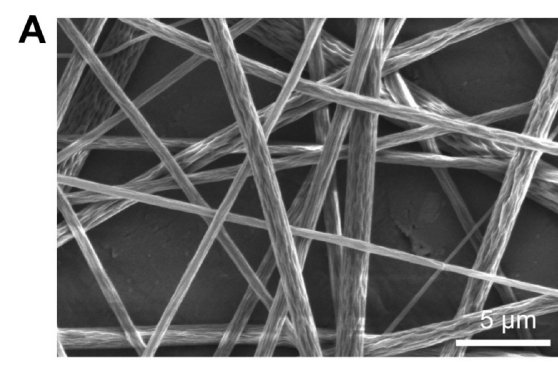

B

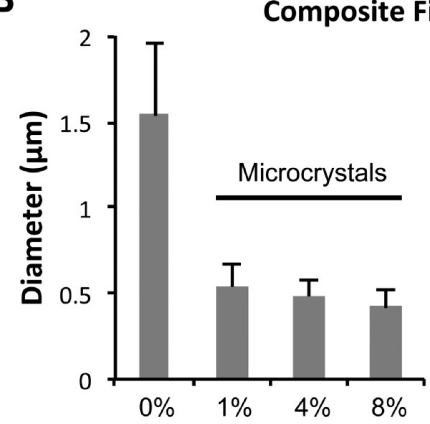

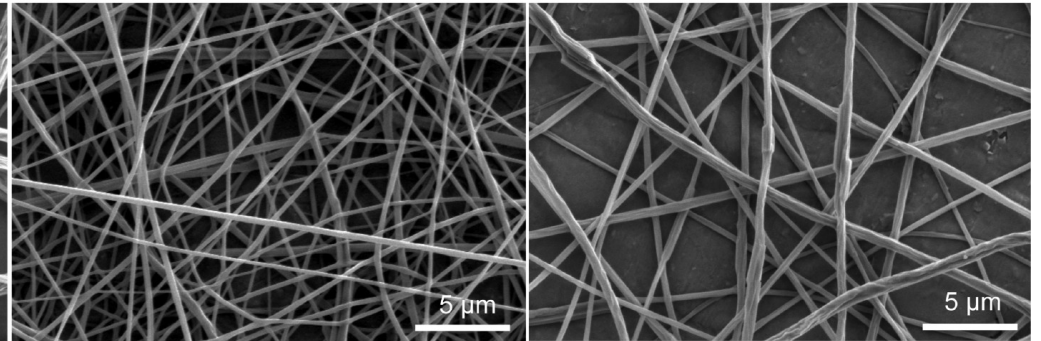

C

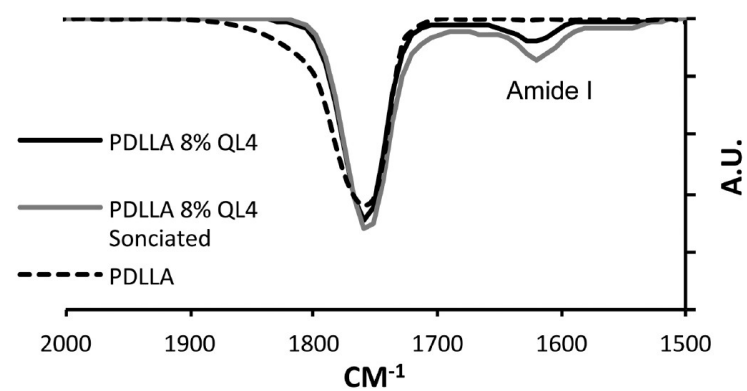

Figure 3. Characterization of composite fibers. (A) Representative SEM images of electrospun fibers with $0 \%$ (left), $8 \%$ pristine (center), and $8 \%$ sonicated (right) DLCP-NTs. (B) Average fiber diameter. (C) FTIR spectra of composite fiber mats in which the dashed line corresponds to pure PDLLA and the black and gray lines correspond to pristine microcrystals and sonicated microcrystals respectively. Amide $\mathrm{C}=\mathrm{O}$ stretch peak near $1620 \mathrm{~cm}^{-1}$ indicates the presence of DLCP-NTs in the fibers.

spinning solutions, little to no beading was observed at an $8 \%$ polymer concentration. As beading can be caused by inefficient polymer entanglement, it is possible that favorable polymermicrocrystal interactions promote increased effective entanglement during the spinning procedure. Like the fibers composed of pure PDLLA, those containing DLCP-NTs exhibited smooth morphologies, suggesting that the microcrystals were aligned with and fully encapsulated by a polymeric sheath (Figure 3A). If the microcrystals exhibited alternative alignments, they would be visibly protruding from the fiber, which is not observed in any of the SEM images. Fibers spun with intact microcrystals consistently exhibited smaller diameters $(\sim 0.5 \mu \mathrm{m})$ compared to those composed of pure PDLLA $(\sim 1.5 \mu \mathrm{m})$ (Figure 3B). This observation is in accordance with previous publications on nanocomposite fibers, which have attributed it to increased conductivity or changes in viscosity of the spinning solution upon addition of the nanofiller. ${ }^{24,25}$ The resultant nanofiber mats were self-standing and sufficiently strong to be lifted from their collection surface for further characterization. The presence of QL4 DLCP-NTs within the fiber was confirmed by Fourier-transform infrared spectroscopy (FTIR) (Figure 3C). The Amide I $\left(1625 \mathrm{~cm}^{-1}\right)$ peak, which is characteristic of proteins and peptides, is present only for fibers containing DLCP-NTs. The IR absorbance peak at $1750 \mathrm{~cm}^{-1}$, which is present in all three samples, corresponds to the carbonyl stretching frequency of the PDLLA matrix. Furthermore, the position of the amide $\mathrm{N}-\mathrm{H}$ stretch at $3278 \mathrm{~cm}^{-1}$ (Figure S5) matches previously reported values, ${ }^{16}$ which were correlated with the presence of a tightly hydrogen bonded network with an interpeptide distance of 4.7-4.8 $\AA$, according to a Krimm's analysis, ${ }^{26}$ indicating that the microcrystals are ordered and composed of nanotubes.

In order to probe the mechanical stabilization conferred by QL4, individual fibers were subjected to nanoindentation experiments using an atomic force microscope with a glass microbead (40 $\mu \mathrm{m}$ diameter) affixed to the cantilever tip
(Figure 4A). Average force displacement curves were generated for dried fibers on silicon backing with the following four fiber

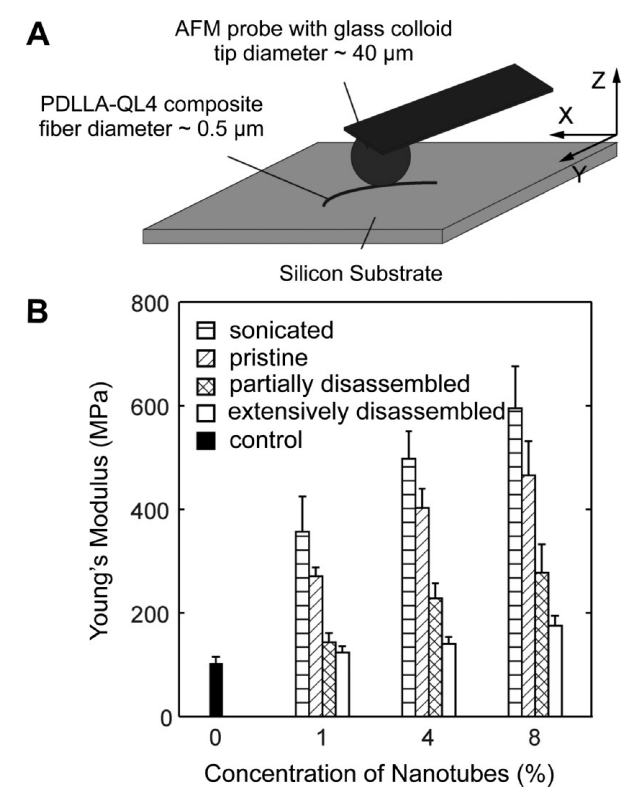

Figure 4. Nanomechanical characterization of composite fibers. (A) Schematic of AFM probe with a spherical colloidal tip used for testing. (B) Average Young's modulus of fibers plotted against DLCP-NT concentration for sonicated, intact, partially disassembled, and extensively disassembled microcrystals $(N=16 ; N=48$ for control).

compositions: $0 \%, 1 \%, 4 \%, 8 \%$ by weight DLCP-NTs (Figure S6). Fibers containing pristine microcrystals were compared to those containing sonicated microcrystals as well as those that had been either partially or extensively disassembled by lyophilization (Figure S2). In order to convert the force versus displacement data to elastic modulus values, we applied the nonlinear Hertzian contact model (Figure S7) ${ }^{27}$ corrected for 
indentation geometry by finite element analysis (Figure S8). Indeed, there is a dose-dependent increase in average fiber modulus, with the highest QL4 loading (8\%) leading to the highest modulus values for each fiber type. However, the wide range of modulus values obtained from individual forcedisplacement experiments on fibers with the highest average stiffness values suggests that the peptides were distributed inhomogeneously within the fiber (Figure S9). Furthermore, we observed significantly decreased reinforcement for the samples in which the DLCP-NTs had been disassembled partially and a further decrease in reinforcement when the DLCP-NTs had been disassembled extensively.

Composite fibers reinforced with sonicated microcrystals exhibited the highest average stiffness values, despite the fact that their average length was smaller than microcrystals in the pristine sample. We believe that this is likely due to their narrower length distribution (Figure 2B), which allows for more homogeneous mixing in the prespinning solution, and consequently more contact area with the surrounding polymer matrix and better stress transfer from microcrystal to polymer after fiber formation. Due to the decrease in the regularity of the structure of disassembled microcrystals, it was not possible to compare their average lengths to the pristine or sonicated sample-sets. Fibers containing sonicated microcrystals at a loading of $8 \%$ exhibited a Young's modulus of $\sim 600 \mathrm{MPa}$, corresponding to a $>5$-fold increase in stiffness over fibers composed of PDLLA alone (see Figures S10 and S11 for a complete table of moduli and statistical significance). It is theoretically possible that variations in fiber diameter across the samples could affect the fiber mechanics. However, based on an analysis of modulus versus fiber diameter for the experimental sample set, it is apparent that the amount and identity of the filler material are the main contributors to the increased stiffness values (Figure S12).

DLCP microcrystals are sufficiently robust to withstand the high electric potentials and high shear forces associated with the electrospinning process. Upon completion of mechanical analysis, the fibers were sacrificed to analyze the integrity of the embedded DLCP microcrystals. Electrospun fiber mats were immersed in acetone to selectively dissolve the PDLLA while leaving the DLCP-NTs unaffected. The recovered microcrystals exhibit a shorter average length than they did before spinning, but their width and overall appearance remains unaltered (Figure S13). This suggests that the spinning process leads to transverse fracture of the longer microcrystals.

\section{CONCLUSION}

In summary, we demonstrated that electrospun PDLLA fibers can be reinforced by incorporation of self-assembled cyclic peptide nanotubes. Higher concentrations of uniform microcrystals led to higher stiffness values, with the stiffest fibers obtained with $8 \%$ by weight loading of sonicated DLCP microcystals exhibiting a modulus value of $600 \mathrm{MPa}$. We also demonstrated that DLCP-NTs are able to withstand the high energy conditions associated with electrospinning, potentially establishing DLCP-NTs as a compatible filler component in a variety of other material processing techniques. Nano- and microstructures assembled from DLCPs possess a unique combination of customizable surface chemistry and rigidity that are absent in most high aspect ratio nanoscale materials. However, examples of DLCP incorporation into macroscopic materials have heretofore been limited. The versatility provided by the self-assembling DLCP structures makes them a potentially useful alternative to those restricted by covalent bonding when considered for applications in high-performance and self-healing materials.

\section{ASSOCIATED CONTENT}

\section{Supporting Information}

The Supporting Information includes a diagram and mass spectrometric characterization of QL4, optical micrographs of QL4 microcrystals at varying degrees of degradation, scanning electron micrographs of composite fibers at varying polymer concentrations, force-displacement curves of single fiber indentations, a Hertzian-contact model describing the indentation of single fibers, finite element analysis of contact geometry, and scanning electron micrographs of extracted QL4 microcrystals from composite fibers. This material is available free of charge via the Internet at http://pubs.acs.org

\section{AUTHOR INFORMATION}

\section{Corresponding Author}

*Mailing address: Pierce Hall, 29 Oxford St., Cambridge, MA 02138; tel: 617-432-7730; e-mail: njoshi@seas.harvard.edu.

\section{Author Contributions}

The manuscript was written through contributions of all authors. All authors have given approval to the final version of the manuscript.

\section{Notes}

The authors declare no competing financial interest.

\section{ACKNOWLEDGMENTS}

This work was funded by a seed grant from the Harvard MRSEC and the Army Research Office (ARO-167836) STIR program.

\section{REFERENCES}

(1) Freed, L. E.; Engelmayr, G. C.; Borenstein, J. T.; Moutos, F. T.; Guilak, F. Adv. Mater. 2009, 21, 3410-3418.

(2) Zahedi, P.; Rezaeian, I.; Ranaei-Siadat, S.-O.; Jafari, S.-H.; Supaphol, P. Polym. Adv. Technol. 2009, 21, 77-95.

(3) Cai, Z.-X.; Mo, X.-M.; Zhang, K.-H.; Fan, L.-P.; Yin, A.-L.; He, C.-L.; Wang, H.-S. Int. J. Mol. Sci. 2010, 11, 3529-3539.

(4) Tian, H.; Tang, Z.; Zhuang, X.; Chen, X.; Jing, X. Prog. Polym. Sci. 2012, 37, 237-280.

(5) Ma, W.; Liu, L.; Zhang, Z.; Yang, R.; Liu, G.; Zhang, T.; An, X.; Yi, X.; Ren, Y.; Niu, Z.; Li, J.; Dong, H.; Zhou, W.; Ajayan, P. M.; Xie, S. Nano Lett. 2009, 9, 2855-2861.

(6) Wang, T.; Drzal, L. T. ACS Appl. Mater. Interfaces 2012, 4, 50795085.

(7) Velasco-Santos, C.; Martinez-Hernandez, A.; Castano, V. Compo. Interfaces 2005, 8, 567-586.

(8) Young, K.; Blighe, F.; Vilatela, J.; Windle, A.; Kinloch, I.; Deng, L.; Young, R.; Coleman, J. ACS nano 2010, 4, 6989-6997.

(9) Dugan, J. M.; Gough, J. E.; Eichhorn, S. J. Nanomedicine 2013, 8, 287-298.

(10) Van Der Zande, M.; Junker, R.; Walboomers, X. F.; Jansen, J. A. Tissue Eng., Part B: Rev. 2011, 17, 57-69.

(11) Boyle, A. L.; Woolfson, D. N. Chem. Soc. Rev. 2011, 40, 4295.

(12) Matson, J. B.; Zha, R. H.; Stupp, S. I. Curr. Opin. Solid State Mater. Sci. 2011, 15, 225-235.

(13) Zhang, S. Nat. Biotechnol. 2003, 21, 1171-1178.

(14) Jung, J.; Gasiorowski, J.; Collier, J. Pept. Sci. 2010, 94, 49-59.

(15) Fernandez-Lopez, S.; Kim, H.-S.; Choi, E. C.; Delgado, M.; Granja, J. R.; Khasanov, A.; Kraehenbuehl, K.; Long, G.; Weinberger, D. A.; Wilcoxen, K. M. Nature 2001, 412, 452-455.

(16) Hartgerink, J.; Granja, J.; Milligan, R.; Ghadiri, M. J. Am. Chem. Soc. 1996, 118, 43-50. 
(17) Diaz, J.; Çağin, T. Nanotechnology 2010, 21, 115703.

(18) Peltoniemi, H.; Ashammakhi, N.; Kontio, R.; Waris, T.; Salo, A.; Lindqvist, C.; Grätz, K.; Suuronen, R. Oral Surg., Oral Med., Oral Pathol., Oral Radiol., Endodontol. 2002, 94, 5-14.

(19) Böstman, O.; Pihlajamäki, H. Biomaterials 2000, 21, 2615-2621.

(20) Felfel, R. M.; Ahmed, I.; Parsons, A. J.; Rudd, C. D. J. Mech. Behav. Biomed. Mater. 2013, 17, 76-88.

(21) Even, N.; Adler-Abramovich, L.; Buzhansky, L.; Dodiuk, H.; Gazit, E. Small 2011, 7, 1007-1011.

(22) McMurray, J. S. Tetrahedron Lett. 1991, 32, 7679-7682.

(23) Hutter, J. L.; Bechhoefer, J. Rev. Sci. Instrum. 1993, 64, 1868.

(24) Changsarn, S.; Mendez, J. D.; Shanmuganathan, K.; Foster, E. J.; Weder, C.; Supaphol, P. Macromol. Rapid Commun. 2011, 32, 13671372.

(25) Liao, G.-Y.; Zhou, X.-P.; Chen, L.; Zeng, X.-Y.; Xie, X.-L.; Mai, Y.-W. Compos. Sci. Technol. 2012, 72, 248-255.

(26) Bandekar, J.; Krimm, S. Biopolymers 1988, 27, 909-921.

(27) Ng, L.; Hung, H.-H.; Sprunt, A.; Chubinskaya, S.; Ortiz, C.; Grodzinsky, A. J. Biomech. 2007, 40, 1011-1023. 